\title{
Histologic evidence for mild lesions in coeliac disease: the challenge is open
}

\author{
Paola Ilaria Bianchi · Federico Biagi • \\ Gino Roberto Corazza
}

Received: 6 April 2011/Accepted: 26 April 2011/Published online: 15 May 2011

(C) SIMI 2011

Even though research on coeliac disease (CD) has already given us fundamental clinical and pathogenic clarifications, $\mathrm{CD}$ is a very complex disease that has not finished surprising us. In the last two decades, the attention of clinicians was focussed on the wide spectrum of clinical presentations of the disease. This has made it possible to diagnose many patients with minor and silent forms of CD, particularly in adulthood, patients who would not have received a diagnosis in the past [1]. In the meanwhile, the incidence and prevalence of CD in Western Countries have increased so greatly that awareness of $\mathrm{CD}$ has increased not only among clinicians, but also among the public and alternative medicine practitioners, often producing a great deal of confusion in the correct criteria for diagnosis $[2,3]$.

In this issue of Internal and Emergency Medicine, Licata and colleagues [4] report on a very hot topic, coeliac patients with only minimal intestinal lesions, and how to diagnose them. Other clinicians have investigated this point $[5,6]$. Among the first, Rostami [5] cast doubts about the great sensitivity of endomysium and gliadin antibodies in the diagnosis of $\mathrm{CD}$ in the cases with only mild lesions found on histology. The accuracy of antibody tests for CD was reviewed by Hill and colleagues [7], who found 95\% mean sensitivity and $99 \%$ mean specificity for IgA endomysial antibodies, and 87 and 95\%, respectively, for IgA tissue transglutaminase antibodies. Obviously, all the serology known for CD was developed using villous atrophy as the gold standard reference, and this can be considered a limit. It became clear that there were some

P. I. Bianchi · F. Biagi $(\bowtie) \cdot$ G. R. Corazza

Coeliac Centre, First Department of Internal Medicine,

Fondazione IRCCS Policlinico San Matteo, University of Pavia,

P.le Golgi, 19, 27100 Pavia, Italy

e-mail: f.biagi@smatteo.pv.it subjects with stigmata of $\mathrm{CD}$, who present positive antibodies many years before developing the enteropathy [8], and these patients were called potentials. Moreover, there are reports of relatives of coeliac patients who have only an increased number of intraepithelial lymphocytes with negative antibodies, but who should also be considered potentials. [9].

On the other hand, the same question regarding the sensitivity of coeliac antibodies in those CD cases without frank atrophy could be put the other way around: are minimal lesions seen on histology specific for CD? Such a question would make the discussion even more complex. We cannot forget that Marsh himself, while classifying coeliac enteropathy histological findings by degree of severity, argues that none of the findings are specific for CD [10]. Many authors describe many alternative causes of intraepithelial lymphocytosis to CD [11-14]. Therefore, it is reasonable to argue that intraepithelial lymphocytosis, like inflammation, is a common pathological finding.

The absence of genetic predisposition can help to exclude CD in borderline case, and a so-called gene-dose effect of HLA-DQ2 in the severity of intestinal atrophy and in the prognosis of refractory $\mathrm{CD}$ has been described. Nevertheless, researchers estimate that the genetic effect attributable to HLA is $53 \%$, agreeing that other non-HLA regions must be involved. Moreover, DQ2 is carried by almost a third of the general population. The cases of mild histological lesions described by Licata and colleagues [4] show a lower predominance of HLA-DQ2/DQ8 genotype. Does this finding open a door to a possible different genetic pattern in this subtype of patients that has not yet been studied? How important is it not to miss a diagnosis of CD? What does a patient do with a potential CD risk? There are very early descriptions of cases of potential $\mathrm{CD}$ in dermatitis herpetiformis patients who underwent intestinal 
lymphoma. Actually, there is a lack of concordance on the increase of mortality in unrecognized CD. Moreover, 2 years of diagnostic and therapeutic delay does not seem to be significant in the development of CD complications [15]. Furthermore, it is still not clear whether it is preferable to start a gluten-free diet immediately, or a careful follow-up, and begin a gluten-free diet only after unequivocal mucosal flattening is recorded. In addition, the mortality rate is not increased in these patients who have only mild symptoms, or of those who have no symptoms at all [15]. However, we should not forget the gradient of mortality in symptomatic coeliac populations from Northern to Southern Europe that particularly affects Sicily [15]. It would be of great interest to study the relative risk of mortality and morbidity in patients with mild intestinal lesions and coeliac negative serology. To date, the only paper on this topic reports a high hazard ratio of mortality for cardiovascular and malignancy causes in Marsh I and II subclass [16]. However, the authors themselves observe that the mortality is increased only during the first year of follow up and that the patients were older or affected by severe comorbidities that made them undergo a greater number of medical examinations. Unfortunately, this study was based on histology records matched with a population health register, and neither distinguishes between positive and negative antibody subjects nor it can provide information about their diet [16].

Licata et al. [4] remind us that nowadays there is a mare magnum of available diagnostic tools for $\mathrm{CD}$, in which only the clinician can make some order and attribute the real weight to each clue, in the individual clinical case. While the diagnosis of CD seems to have become easier over the years, the facilities are a double-edged weapon: the diagnosis of $\mathrm{CD}$ is still challenging.

In the absence of a univocal diagnostic test able not only to predict the presence of $\mathrm{CD}$, but also the possible future of the disease, the importance of avoiding a hasty diagnosis of CD should be emphasized, since that diagnosis implies the start of what may be a useless gluten-free diet, and may obscure the possibility of making the correct diagnosis. In Italy, coeliac patients are guaranteed considerable economic benefits by the National Health Service. This means that a wrong diagnosis would burden not only the individual patients, but also the community. The wait-and-see strategy is probably still the most prudent and best one.
Conflict of interest None.

\section{References}

1. Biagi F, Corazza GR (2002) Clinical features of coeliac disease. Dig Liver Dis 34:225-228

2. Biagi F, Klersy C, Balduzzi D, Corazza GR (2010) Are we not over-estimating the prevalence of coeliac disease in the general population? Ann Med 42:557-561

3. Biagi F, Bianchi PI, Campanella J, Zanellati G, Corazza GR (2009) The impact of misdiagnosing celiac disease at a referral centre. Can J Gastroenterol 23:543-545

4. Licata A, Cappello M, Arini A, Florena AM, Randazzo C, Butera $\mathrm{G}$ et al (2011) Serology in adults with celiac disease: limited accuracy in patients with mild histological lesions. Intern Emerg Med (present issue). doi:10.1007/s11739-011-0585-8

5. Rostami K, Kerckhaert J, Tiemessen R, von Blomberg ME, Meijer JWR, Mulder CJJ (1999) Sensitivity of antiendomysium and antigliadin antibodies in untreated celiac disease: disappointing in clinical practice. Am J Gastroenterol 94:888-894

6. Dickey W, Hughes DR, McMillan SA (2000) Reliance on serum endomysial antibody testing underestimates the true prevalence of coeliac disease by one fifth. Scand J Gastroenterol 35:181-183

7. Hill ID (2005) What are the sensitivity and specificity of serologic test for celiac disease? Do sensitivity and specificity vary in different populations? Gastroenterology 128:S25-S32

8. Collin P, Helin H, Mäki M, Hällström O, Karvonen AL (1993) Follow up of patients positive in reticulin and gliadin antibody tests with normal small-bowel biopsy findings. Scand J Gastroenterol 28:595-598

9. Mäki M, Holm K, Collin P, Savilahti E (1991) Increase in $\gamma / \delta$ T cell bearing lymphocytes in normal small bowel mucosa in latent coeliac disease. Gut 32:1412-1414

10. Marsh MN, Crowe PT (1995) Morphology of the mucosal lesion in gluten sensitivity. Bailliere's Clin Gastroenterol 9:273-293

11. Katz AJ, Grand RJ (1979) All the flattens is not "sprue". Gastroenterology 76:375-377

12. Goldstein NS (2004) Non-gluten sensitivity-related small bowel villous flattening with increased intraepithelial lymphocytes: not all that flattens is celiac sprue. Am J Clin Pathol 121:546-550

13. Lähdeaho ML, Kaukinen K, Collin P, Ruusta T, Partanen J, Haapla AM, Mäki M (2005) Celiac disease: from inflammation to atrophy: a long-term follow up study. J Pediatr Gastroenterol Nutr 41:44-48

14. Biagi F, Bianchi PI, Campanella J, Badulli C, Martinetti M, Klersy C et al (2008) The prevalence and the causes of minimal intestinal lesions in patients complaining of symptoms suggestive of enteropathy: a follow up study. J Clin Pathol 61:1116-1118

15. Biagi F, Corazza GR (2010) Mortality in celiac disease. Nat Rev Gastroenterol Hepatol 7:158-162

16. Ludvigsson JF, Montgomery SM, Ekbom A, Brandt L, Granath F (2009) Small-intestinal histopathology and mortality risk in celiac disease. JAMA 302:1171-1178 